IBD

\section{OTU-001 IDENTIFICATION OF A NOVEL THERAPEUTIC AGENT FOR TREATING IBD GUIDED BY SYSTEMS MEDICINE}

1,2Michael Burkitt*, ${ }^{2}$ Kate Lloyd, ${ }^{2,3}$ Stamatia Papoutsopoulou, ${ }^{3}$ Emily Smith, ${ }^{4}$ Philip Stegmaier, ${ }^{2} \mathrm{D}$ Mark Pritchard, ${ }^{4}$ Alexander Kel, ${ }^{3}$ Werner Müller, ${ }^{2}$ Chris Probert. ${ }^{1}$ Manchester University NHS Foundation Trust, Manchester, UK; ${ }^{2}$ University of Liverpool, Liverpool, UK; ${ }^{3}$ University of Manchester, Manchester, UK; ${ }^{4}$ GeneXplain GmbH, Wolfenbüttel, Germany

\subsection{6/gutjnl-2018-BSGAbstracts.104}

Introduction There remains an unmet need in the treatment of IBD. The SysmedIBD project established a multi-disciplinary consortium to systematically investigate patients with inflammatory bowel disease, focusing on the dynamics of NF- $\mathrm{B}$ signalling. Through this approach we identified an established drug with potential for repurposing to treat IBD, in selected patients.

Methods Novel targets with potential for impacting outcomes of IBD were identified in-silico by combining integrated promoter/pathway analysis of published microarray data and systematic text-mining of the published literature using the geneXplain software platform. An established drug with potential for repurposing was assessed as a proof-of-concept agent using a multi-step validation pipeline based on its effect on NF- $\mathrm{BB}$ dynamics in-vitro and in-vivo, and its ability to ameliorate murine experimental colitis.

Results 3191 pharmacological agents (Prestwick Chemical Library) were assessed in-silico. 36 agents were highly significantly predicted to influence NF- $\kappa \mathrm{B}$ and other IBD target activity. Amongst the highest ranked agents were the macrolide antibiotics. Clarithromycin (CLA) was selected as a paradigm for subsequent analyses.

The effects of CLA were investigated in 5 experiments:

1. $\mathrm{NF}-\kappa \mathrm{B}$ mediated transcription was investigated using peritoneal macrophages and enteric organoids from a mouse expressing firefly luciferase under the control of the human TNF promoter: CLA suppressed responses in both tissues $(\mathrm{p}<0.05)$.

2. $\mathrm{NF}-\kappa \mathrm{B}(\mathrm{p} 65)$ protein shuttling dynamics were characterised in enteric organoids cultured from a mouse expressing human p65-dsRed: CLA suppressed TNF induced oscillation of p65 $(\mathrm{p}=0.0002)$.

3. C57BL/6 mice were treated with intra-peritoneal LPS $(0.125 \mathrm{mg} / \mathrm{kg})$ to induce small intestinal $\mathrm{NF}-\kappa \mathrm{B}$ activation:

CLA suppressed DNA binding of $\mathrm{p} 65(\mathrm{p}=0.002)$.

4. The effect of CLA on DSS colitis was studied: mice treated with CLA lost significantly less weight $(\mathrm{p}<0.05)$, and had less severe histology than mice treated with vehicle $(p=0.004)$.

5. The effect of CLA on TNF induced nuclear localisation of p65 in human enteric organoids was studied: CLA suppressed p65 nuclear localisation $(\mathrm{p}<0.0001)$.

Conclusions Using a systems biology approach, we have identified an agent with potential for repurposing to treat IBD. Outcomes of earlier clinical trials of clarithromycin were discordant: we are developing a biomarker of NF- $\mathrm{BB}$ responsiveness that may enable precise selection of patients for a personalised medicine trial.
OTU-002 HLA-DQA1 CONTRIBUTES TO THE DEVELOPMENT OF ANTIBODIES TO ANTI-TNF THERAPY IN CROHN'S DISEASE

${ }^{1}$ Aleksejs Sazonovs, ${ }^{2}$ Nicholas A Kennedy*, ${ }^{2}$ Claire Bewshea, ${ }^{1}$ Loukas Moutsianas, ${ }^{2}$ Gareth J Walker, 'Katrina De Lange, ${ }^{2}$ James R Goodhand, ${ }^{1}$ Carl Anderson, 'Jeff Barrett, PANTS investigator consortium, ${ }^{2}$ Tariq Ahmad. ${ }^{1}$ Wellcome Trust Sanger Institute, Hinxton, UK; ${ }^{2} I B D$ Pharmacogenetics, University of Exeter, Exeter, UK

\subsection{6/gutjnl-2018-BSGAbstracts. 105}

Background Immunogenicity to anti-TNF therapy is a major cause of loss of response, treatment discontinuation and hypersensitivity reactions and currently cannot be predicted prior to treatment. A number of factors have been associated with the risk of immunogenicity, but knowledge of the cellular and molecular mechanisms remain limited. Our aim was to investigate genetic susceptibility to immunogenicity.

Methods The PANTS (Personalised Anti-TNF Therapy in Crohn's disease) study is a 3 year prospective observational UKwide study investigating primary non-response, loss of response and adverse drug reactions to the anti-TNF drugs infliximab and adalimumab. Anti-drug antibodies (ADAs) were measured serially at trough using the IDKmonitor total ADAb ELISA assay. Immunogenicity was defined as (a) ADA titre $\geq 10 \mathrm{AU} / \mathrm{ml}$ and (b) ADA titre $\geq 10 \mathrm{AU} / \mathrm{ml}$ with no detectable drug. A genomewide association study (GWAS) was carried out on imputed genotype data using a Cox proportional hazards model incorporating the anti-TNF used and presence of concomitant immunomodulator as covariates (SurvivalGWAS_SV v1.3.1).

Results After quality control, we had genotype data for 1284 patients followed prospectively for a minimum of 12 months since starting anti-TNF therapy. Using a Cox proportional hazards model and an immunogenicity definition of ADAs titre $\geq 10 \mathrm{AU} / \mathrm{ml}$ we identified a genome-wide association on chromosome 6 (top SNP rs74291249 with $\mathrm{p}=5.6 \times 10^{-13}$ ). We imputed the HLA alleles at 2- and 4-digit resolution using the HIBAG package and demonstrated that this signal was driven by HLA-DQA1*05 for both infliximab and adalimumab. No additive effect of having two DQA1*05 was seen. Figures 1 and 2 show immunogenicity-free survival stratified by HLA-DQA1*05 genotype and concomitant immunomodulators at baseline.

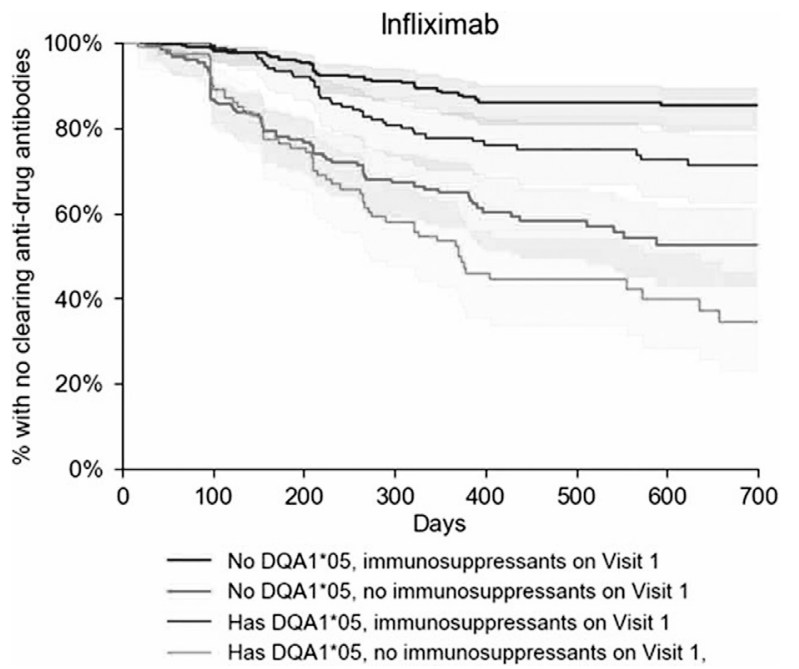

Abstract OTU-002 Figure 1 Immunogenicity by immunomodulator and HLA-DQA1*05 for infliximab and adalimumab. 


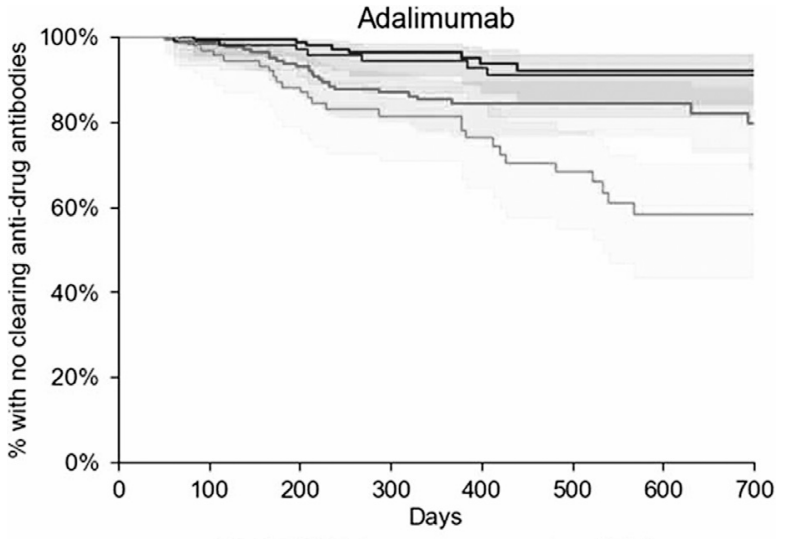

- No DQA1*05, immunosuppressants on Visit 1

- No DQA1 ${ }^{\circ} 05$, no immunosuppressants on Visit 1

- Has DQA ${ }^{*} 05$, immunosuppressants on Visit 1

- Has DQA1*05, no immunosuppressants on Visit 1

\section{Abstract OTU-002 Figure 2}

Conclusion We have demonstrated that immunogenicity to anti-TNF is determined by HLA variants. Pre-treatment genetic testing might allow the use of individual risk profiles and targeted use of immunomodulatory therapies to deliver more durable, safe and cost-effective anti-TNF therapy.

\section{OTU-003 ETROLIZUMAB AS INDUCTION THERAPY IN MODERATE TO SEVERE CROHN'S DISEASE: RESULTS FROM BERGAMOT COHORT 1}

\begin{abstract}
${ }^{1}$ Christian Selinger*, ${ }^{2}$ William Sandborn, ${ }^{3} J u l i a n$ Panes, ${ }^{4,5}$ Jennifer Jones, ${ }^{6}$ Azra Hassanali, ${ }^{7}$ Rhian Jacob, ${ }^{6}$ Zaineb Sharafali, ${ }^{6}$ Young Oh, ${ }^{6}$ Swati Tole. 'Leeds Teaching Hospitals Nhs Trust, Leeds, UK; ${ }^{2}$ University of California, La Jolla, USA; ${ }^{3}$ University of Barcelona, Barcelona, Spain; ${ }^{4}$ Queen Elizabeth II Health Sciences Center, Halifax, Canada; ${ }^{5}$ Dalhousie University, Halifax, Canada; ${ }^{6}$ Genentech, Inc, San Francisco, USA; ${ }^{7}$ Roche Products Ltd, Welwyn Garden City, UK
\end{abstract}

\subsection{6/gutjnl-2018-BSGAbstracts. 106}

Introduction Etrolizumab (etro), a humanised anti- $\beta 7$ monoclonal antibody was evaluated in patients (pts) with moderate to severe Crohn's Disease (CD) to determine safety and efficacy. Results of the Phase III BERGAMOT (NCT02394028) exploratory induction cohort are presented.

Methods Eligible pts with moderate to severe CD (refractory/ intolerant to anti-TNF $\alpha$ agents, immunosuppressants, and/or corticosteroids) were assigned (2:2:1) etro $105 \mathrm{mg}$ SC Q4W, etro $210 \mathrm{mg}$ at wks $0,2,4,8$, and 12 , or placebo (pbo) during a 14-wk induction period. Endpoints included CDAI remission (CDAI <150), CDAI-100 and -70 responses, PRO2 remission (weighted combined score $\leq 11$, based on pt report of liquid/very soft stool frequency [SF] and abdominal pain [AP]), symptomatic remission (unweighted $\mathrm{SF} \leq 3$ and $\mathrm{AP} \leq 1$ ), and endoscopic improvement $(\geq 50 \%$ reduction from baseline SES-CD) at wk 14.

Results 300 pts (73\% aTNF-experienced) with moderate to severe CD (mean CDAI [SD], 315.6 [60.0]; mean SES-CD [SD], 14.1 [7.3]; median faecal calprotectin [range] 918 [30$15451] \mu \mathrm{g} / \mathrm{g}$; median C-reactive protein [range], 9.75 [0.2$148.0] \mathrm{mg} / \mathrm{L})$ received etro $105 \mathrm{mg}(\mathrm{n}=120)$, etro $210 \mathrm{mg}$ $(n=121)$, or pbo $(n=59)$.

Symptomatic remission was seen in a greater proportion of pts receiving etro $105 \mathrm{mg}$ and $210 \mathrm{mg}$ compared with pbo at wks 6, 10, and 14. More pts achieved endoscopic improvement with etro $105 \mathrm{mg}$ and $210 \mathrm{mg}$ compared with pbo at wk 14 (table 1).

CDAI remission at wk 14 was greater with etro $105 \mathrm{mg}$ and $210 \mathrm{mg}$ compared with pbo: $23.3 \%$ (17.6, 30.2), $28.9 \%$ $(22.7,36.1)$ and $16.9 \%(10.4,26.4)$ respectively. PRO2 remission was achieved at wk 14 in $28.3 \%(22.1,35.5), 28.9 \%$ $(22.7,36.1)$, and $20.3 \%(13.1,30.2)$ of pts, respectively.

The frequency of adverse events with etro was comparable with pbo; no deaths, anaphylaxis or progressive multifocal leucoencephalopathy occurred.

\begin{tabular}{|c|c|c|c|}
\hline \multirow{2}{*}{\multicolumn{2}{|c|}{$\%(90 \% \mathrm{Cl})$}} & Etro $105 \mathrm{mg} \mathrm{n}=120$ & Etro $210 \mathrm{mg} \mathrm{n}=121$ \\
\hline & & & \\
\hline \multicolumn{4}{|c|}{ Symptomatic remission } \\
\hline Wk 6 & $8.5(4.2-16.4)$ & $15.0(10.4-21.1)$ & $25.6(19.7-32.6)$ \\
\hline Wk 10 & $8.5(4.2-16.4)$ & $15.8(11.1-22.1)$ & $27.3(21.2-34.4)$ \\
\hline Wk 14 & $11.9(6.6-20.5)$ & $20.8(15.4-27.5)$ & $24.8(18.9-31.8)$ \\
\hline \multicolumn{4}{|c|}{ Endoscopic improvement } \\
\hline Wk 14 & $3.4(1.1-9.7)$ & $21.0(15.6-27.8)$ & $17.4(12.4-23.7)$ \\
\hline
\end{tabular}

Conclusion Treatment with etro was well tolerated and resulted in clinically meaningful endoscopic improvement in pts with moderate to severe CD. Rapid symptomatic remission was observed as early as wk 6 and sustained through wk 14 . Enrolment into induction cohorts and the maintenance phase is ongoing.

\section{OTU-004 SHALLOW WHOLE-GENOME SEQUENCING PREDICTS THE FUTURE CANCER RISK OF LOW-GRADE DYSPLASTIC LESIONS IN ULCERATIVE COLITIS}

\footnotetext{
1,2Ibrahim Al Bakir*, 'Kathleen Curtius, ${ }^{1}$ Anne-Marie Baker, ${ }^{1}$ Theo so Clarke, ${ }^{3}$ Morgan Moorghen, ${ }^{4}$ Marnix Jansen, ${ }^{4}$ Manuel Rodriguez-Justo, ${ }^{5}$ Simon Leedham, ${ }^{2}$ Ailsa Hart, ${ }^{1}$ Trevor Graham. ${ }^{1}$ Barts Cancer Institute, London, UK; ${ }^{2} I B D$ Unit, St. Mark's Hospital, Harrow, UK; ${ }^{3}$ Pathology Department, St. Mark's Hospital, Harrow, UK; ${ }^{4}$ Pathology Department, University College Hospital, London, UK; ${ }^{5}$ Wellcome Trust Centre for Human Genetics, Oxford, UK
}

\subsection{6/gutjnl-2018-BSGAbstracts. 107}

Background The management of low grade dysplasia (LGD) in ulcerative colitis (UC) is uncertain due to the variable risk of progression to colorectal cancer (CRC). Chromosomal copy number alterations (CNAs) occur in colonic epithelial cells of UC patients who have developed CRC. The burden of CNAs in precursor LGD relative to high-grade dysplasia (HGD) and CRC has not been defined, and the correlation between LGD CNA burden and future HGD/CRC risk is unknown.

Shallow whole-genome sequencing is a novel, cost-effective technique for high resolution CNA assessment in formalinfixed, paraffin-embedded tissue.

Methods We identified 19 UC proctocolectomy specimens with HGD/CRC, and analysed 77 neoplastic regions (36 LGD, $34 \mathrm{HGD}$ and $7 \mathrm{CRC}$ ). We then analysed 13 'progressor' patients with 27 LGD lesions who subsequently developed HGD/CRC a median 427 days later (IQR 213-777), and 22 'non-progressor' patients with 26 LGD lesions who remained 